\title{
A novel comprehensive program combined optimal medical treatment and lifestyle for type 2 diabetes
}

\author{
Chunsong $\mathrm{Hu}^{1}$, Tengiz Tkebuchava ${ }^{2}$, and Qinghua $\mathrm{Wu}^{3}$ \\ ${ }^{1}$ Nanchang University \\ ${ }^{2}$ Office of the President/CEO, Boston TransTec, LLC, Boston \\ ${ }^{3}$ Department of Cardiology, the Second Affiliated Hospital of Nanchang University
}

October 26, 2021

\begin{abstract}
This article introduces briefly current status in managing type 2 diabetes (T2D) and an updated classical standardized comprehensive program which combines optimal medical treatment (OMT) with lifestyle modification, that is, intervention of RT-ABCDEFG (iRT-ABCDEFG) for control and prevention of T2D, and discusses its advantages and prospects. Here, G means goals; F means follow-up; E means examination; D means disease \& risk factors control; C means changing unhealthy "environment-sleep-emotion-exercise-diet" intervention [E(e)SEEDi] lifestyle \& Chinese medicine or control the source of infection \& cutting genetic or spreading pathways during the COVID-19 pandemic; B means biohazard control; And A means antagonistic treatment, such as optimal anti-diabetic agents, the glucagon-like peptide-1 receptor (GLPR) agonists, the sodiumglucose cotransporter 2 (SGLT2) inhibitors, and the ultralong-acting, once-daily basal insulin. As a novel strategy for Intervention of diabetes, this program can be used as a Reverse, Right, and Routine Treatment in clinical practice. Moreover, the vital goals which include less major adverse cardiocerebrovascular events (MACCE) and diabetic complications, less medical costs, longer life expectancy, lower morbidity and mortality, and higher quality of life, will be realized by consistently practicing this program due to early diagnosis, OMT, and overall prevention. Whatever, this program is very helpful to manage or self-manage T2D and improve its outcomes since it highly links to cardiovascular disease (CVD), stroke, cancer, and other MACCEs.
\end{abstract}

\section{Hosted file}

20211019 T2D.doc available at https://authorea.com/users/443069/articles/543140-a-novelcomprehensive-program-combined-optimal-medical-treatment-and-lifestyle-for-type-2diabetes 